\title{
The role of cuticular pheromones in courtship conditioning of Drosophila males
}

\author{
Kathleen K. Siwicki, ${ }^{1,3}$ Paul Riccio, ${ }^{1}$ Lisa Ladewski, ${ }^{1}$ Fabrice Marcillac, ${ }^{2}$ \\ Laurence Dartevelle, ${ }^{2}$ Stephanie A. Cross, ${ }^{1}$ and Jean-François Ferveur ${ }^{2}$ \\ ${ }^{1}$ Department of Biology, Swarthmore College, Swarthmore, Pennsylvania 19081, USA; ${ }^{2}$ Centre National de la Recherche \\ Scientifique, UMR-5548, Insect Development and Chemical Communication, Universite de Bourgogne, Dijon, France
}

\begin{abstract}
Courtship conditioning is an associative learning paradigm in Drosophila melanogaster, wherein male courtship behavior is modified by experience with unreceptive, previously mated females. While the training experience with mated females involves multiple sensory and behavioral interactions, we hypothesized that female cuticular hydrocarbons function as a specific chemosensory conditioned stimulus in this learning paradigm. The effects of training with mated females were determined in courtship tests with either wild-type virgin females as courtship targets, or with target flies of different genotypes that express distinct cuticular hydrocarbon $(\mathrm{CH})$ profiles. Results of tests with female targets that lacked the normal $\mathrm{CH}$ profile, and with male targets that expressed typically female $\mathrm{CH}$ profiles, indicated that components of this $\mathrm{CH}$ profile are both necessary and sufficient cues to elicit the effects of conditioning. Results with additional targets indicated that the female-specific 7,11-dienes, which induce naive males to court, are not essential components of the conditioned stimulus. Rather, the learned response was significantly correlated with the levels of 9-pentacosene (9-P), a compound found in both males and females of many Drosophila strains and species. Adding 9-P to target flies showed that it stimulates courting males to attempt to copulate, and confirmed its role as a component of the conditioned stimulus by demonstrating dose-dependent increases in the expression of the learned response. Thus, 9-P can contribute significantly to the conditioned suppression of male courtship toward targets that express this pheromone.
\end{abstract}

Neural mechanisms that are responsible for behavioral plasticity in Drosophila melanogaster have been addressed primarily in the context of a classical conditioning paradigm in which flies learn to associate electric shocks with olfactory cues (Tully and Quinn 1985). As a result of intensive investigations involving diverse experimental approaches and levels of analysis, a detailed model has evolved of the essential neural substrates and processes underlying this form of olfactory classical conditioning (Davis 1996; Roman and Davis 2001; Waddell and Quinn 2001; Dubnau et al. 2003). A more complex associative learning paradigm is experience-dependent courtship conditioning, wherein male courtship behavior is modified by experience with an unreceptive, previously mated female (Siegel and Hall 1979; Tompkins et al. 1983; McBride et al. 1999). In spite of the ecological relevance of this form of learning, progress in deciphering its neural mechanisms has been impeded by its complexity, specifically by the multisensory nature of the training experience with mated females (Siwicki and Ladewski 2003).

In his first encounter with a potential mate, a male fruit fly follows the target, taps her cuticle with his foretarsi, and vibrates one wing to generate a courtship song (Hall 1994; Greenspan and Ferveur 2000). If the target decreases her locomotion (Tompkins et al. 1982), the male approaches from the rear, extends his proboscis to contact her posterior abdomen, and attempts to copulate (Bastock and Manning 1955; Hall 1994). Socially naive males court not only virgin females, but also unreceptive targets like previously mated females (Bastock and Manning 1955; Connolly and Cook 1973). Experience with mated females results in a form of associative learning called conditioned courtship suppression

${ }^{3}$ Corresponding author.

E-mail ksiwick1@swarthmore.edu; fax (610) 328-8663.

Article published online ahead of print. Article and publication date are at http://www.learnmem.org/cgi/doi/10.1101//m.85605. or courtship conditioning (Siegel and Hall 1979; Tompkins et al. 1983). After $30 \mathrm{~min}$ of courting a mated female, males suppress their courtship toward virgin females for about $2 \mathrm{~h}$ (Siegel and Hall 1979; Kamyshev et al. 1999). Behavioral evidence suggests that this is a form of classical conditioning, in which males learn to associate some female aphrodisiac cue(s) (a conditioned stimulus) with an aversive signal presented by mated females (Tompkins et al. 1983; Ackerman and Siegel 1986). A significant challenge in deciphering the mechanisms of this intriguing form of associative learning has been to identify the relevant stimuli and sensory systems that transduce them.

A requirement for visual stimuli was eliminated by experiments with visually impaired males (Joiner and Griffith 1997), and by successful conditioning of males in the dark or in dim red light (Tompkins et al. 1983; Joiner and Griffith 1997). The importance of chemosensory cues was demonstrated by manipulating male exposure to organic extracts of mated females (Tompkins et al. 1983). Simultaneous exposure to mated-female extract and another fly (even an immobilized male) resulted in a profound suppression of subsequent courtship toward virgins (Tompkins et al. 1983). Either stimulus alone or sequential presentations had no effect, strongly suggesting an associative learning process. In similar experiments, males were successfully conditioned by extracts of virgin females paired with aversive odorants like quinine or cis-vaccenyl acetate (Ackerman and Siegel 1986; Zawistowski 1988). These studies showed that pairing an aversive chemosensory stimulus with organic extracts of females can produce a conditioned suppression of male courtship toward virgins. In the present study, the specific identity of compounds that function as the conditioned stimulus during training with mated females has been addressed by manipulating the pheromonal profiles of flies used to test courtship memory.

Courting males sample the pheromones of target flies via 
olfactory sensilla on the antennae and maxillary palps and gustatory sensilla on the foretarsi and proboscis (Stocker and Gendre 1989; Stocker 1994; Bray and Amrein 2003). Long-chain hydrocarbons on the cuticle of D. melanogaster are sexually dimorphic and include substances that can either stimulate or inhibit male courtship, suggesting a role as sex pheromones (Jallon 1984; Cobb and Ferveur 1996; Ferveur 1997). Recent evidence for the aphrodisiac and anti-aphrodisiac properties of these cuticular compounds derives from genetic manipulations of the hydrocarbons of living courtship targets. Specifically, UAS-transformer (UAS-tra) in combination with various GAL4 drivers can dramatically alter the cuticular hydrocarbon $(\mathrm{CH})$ profiles of both male and female flies (Ferveur and Sureau 1996; Savarit et al. 1999; Savarit and Ferveur 2002a). We have used these pheromonally altered genotypes to test the hypothesis that cuticular pheromones expressed by females are the specific chemosensory cues that function as a conditioned stimulus in courtship conditioning. Moreover, by evaluating individual components of the $\mathrm{CH}$ profile for possible correlations with learning and memory, we have identified 9-pentacosene (9-P) as the most important chemosensory cue for eliciting a learned response from trained males. By adding pure 9-P to test flies, we discovered that it stimulates males to attempt to copulate, and that males are less responsive to this aphrodisiac after training with mated females. The results provide direct evidence for the aphrodisiac properties of 9-P and for the identity of the conditioned stimulus responsible for changes in male courtship behavior following experience with mated females.

\section{Results}

\section{Expression of courtship memory is correlated with cuticular hydrocarbons of the courtship targets}

To test the hypothesis that female cuticular pheromones are the specific chemosensory cues that function as the conditioned stimulus in courtship conditioning, the effects of training with mated females were tested in courtship assays with target flies bearing distinct cuticular hydrocarbon $(\mathrm{CH})$ profiles. The hypothesis predicts that trained males will be less active courters than naive males only with test targets that express the relevant chemosensory cues.

The levels of 24 compounds identified in cuticular extracts (Table 1) of individual flies of the target genotypes were measured by gas chromatography (Antony and Jallon 1982; Savarit and Ferveur 2002a). The identified compounds include both saturated and unsaturated $\mathrm{CHs}$, with chain-lengths of 23C, 25C, 27C, and 29C (Table 1; Fig. 1). CH profiles for many of these genotypes have been reported previously (Antony and Jallon 1982; Jallon and David 1987; Ferveur et al. 1997; Savarit and Ferveur 2002a) and were confirmed for the strains used in this study. The $\mathrm{CH}$ profile of Canton-S (CS) virgin females is characterized by high levels of unsaturated compounds (Fig. 1A, black bars). The predominant components are 7,11-heptacosadiene (7,11-HD, bar \#14) and 7,11-nonacosadiene (7,11-ND, bar \#21), with two double bonds and chain lengths of $27 \mathrm{C}$ and 29C, respectively. These dienes are not expressed by wild-type males (Fig. 1B; Antony et al. 1985; Ferveur and Sureau 1996). They

Table 1. Cuticular hydrocarbons (CHs) identified in hexane extracts of flies used as courtship targets ${ }^{a}$

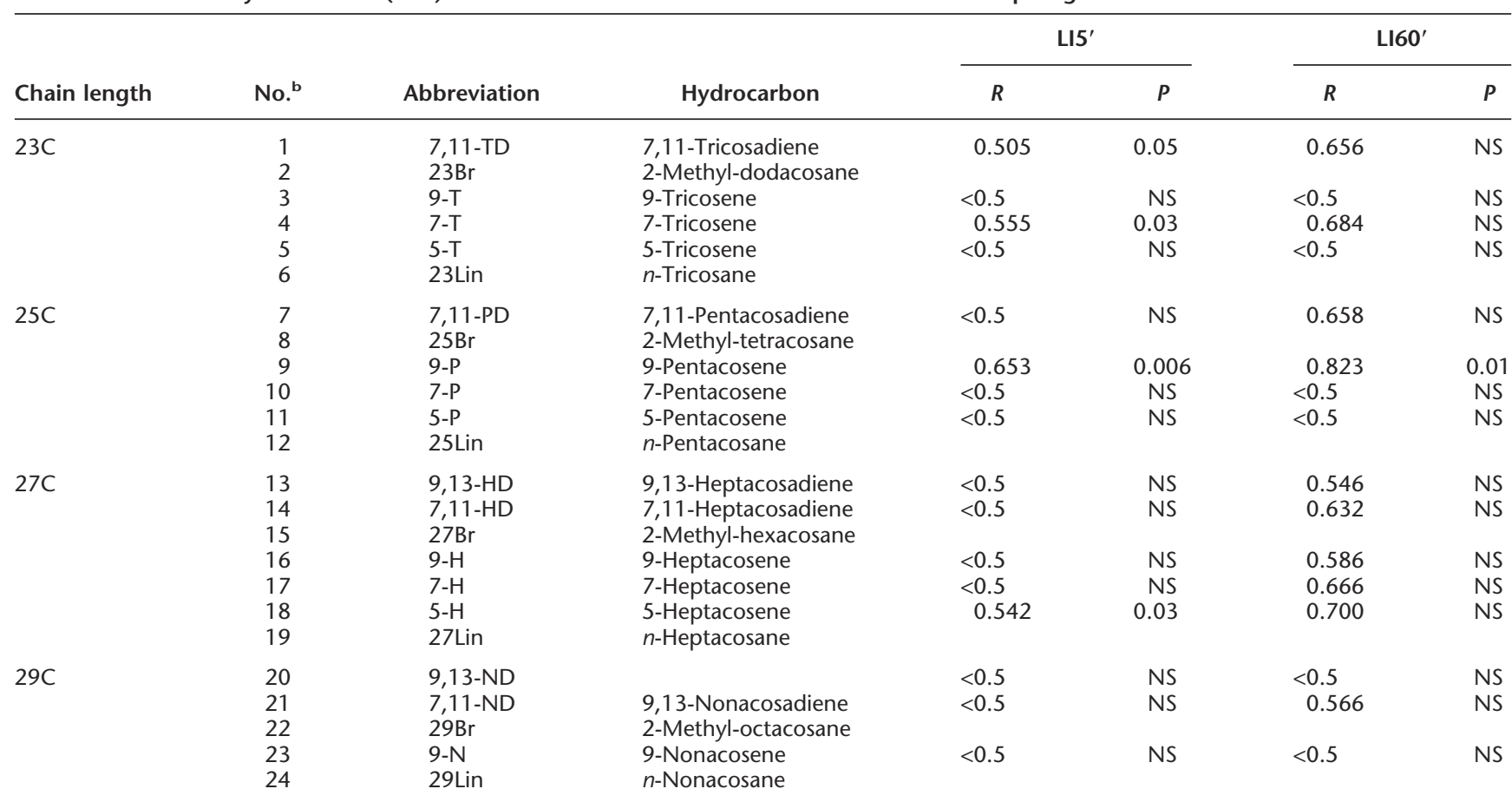

'Data in the four columns on the right describe correlations between Learning Indexes (LI5' and LI60') determined in tests with targets of different genotypes and the levels of 16 unsaturated hydrocarbons in cuticular extracts of the target flies. Six of these correlations are shown as scatterplots in Figure 4. A total of $16 \mathrm{LIS}^{\prime}$ values were determined independently in tests of trained and naive males with nine different target genotypes. The LI60' data set comprised eight values determined independently in tests with seven different target genotypes. The indicated correlation coefficients $(R)$ and $P$-values were determined by simple regressions (without correcting for multiple comparisons). NS indicates $P>0.05$. By using Monte Carlo simulations to evaluate the significance of correlations determined in multiple independent comparisons (see Materials and Methods), 9-P is the only hydrocarbon with which $\mathrm{LI} 5^{\prime}$ is significantly correlated $(P<0.05)$.

${ }^{\text {b}}$ Refers to the bar graphs in Figure 1, depicting the levels of each $\mathrm{CH}$ measured in extracts of individual flies of different genotypes. 

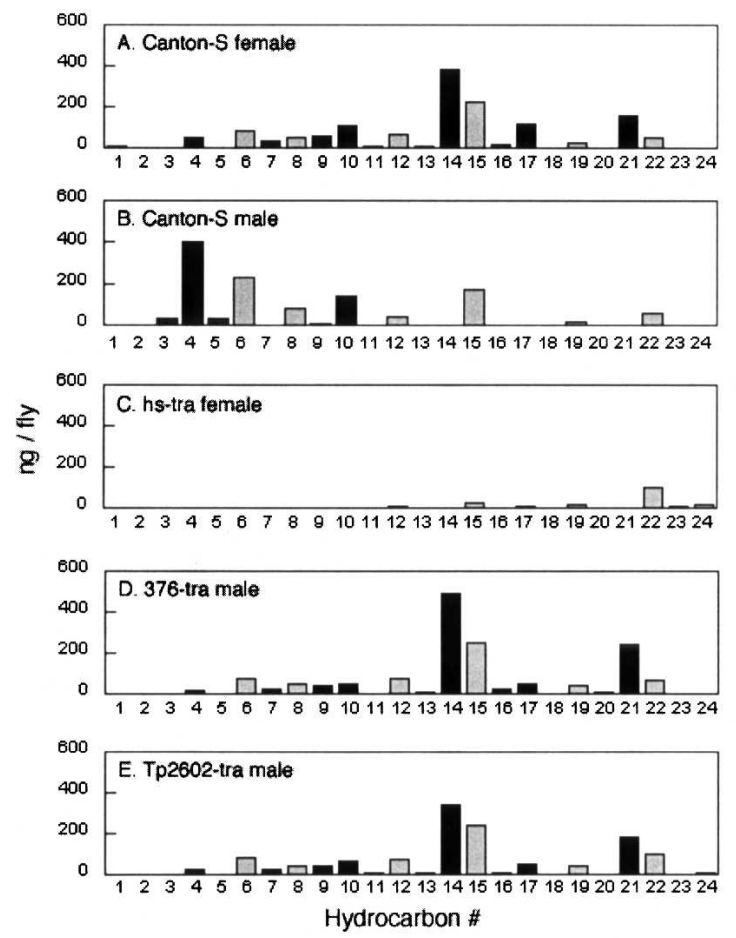
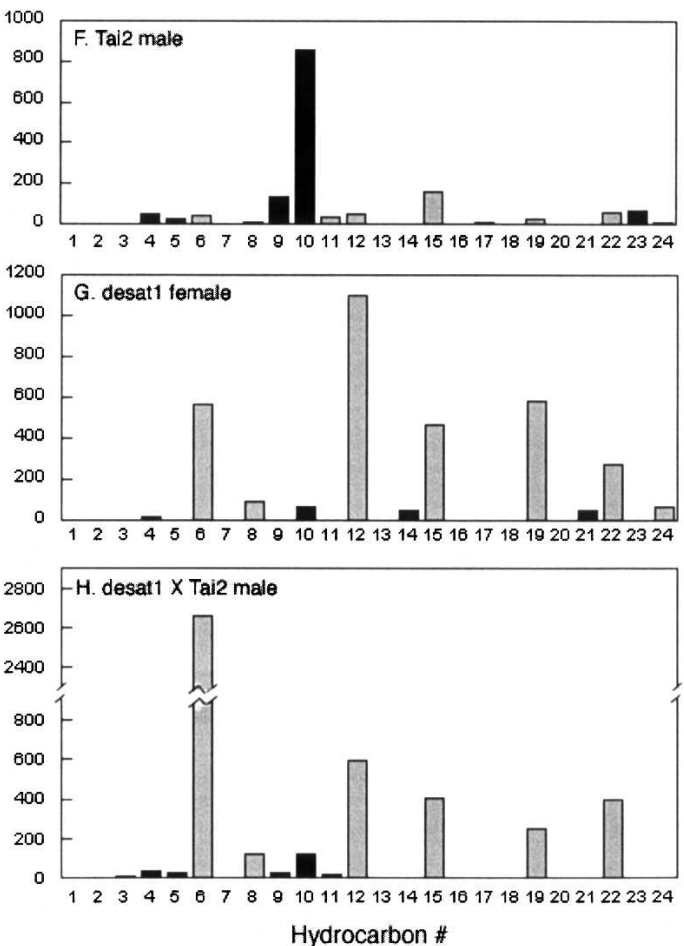

Figure 1. Levels of the 24 most abundant hydrocarbons measured by gas chromatography in cuticular extracts of $(A)$ Canton-S virgin females, $(B)$ Canton-S males, and $(\mathrm{C}-H)$ six different genotypes used as courtship targets to assay the effects of courtship conditioning. The specific compounds represented by each bar number (\#) are identified in Table 1. Data from 6-12 individual flies were averaged for each panel; there was very little variation within each of these genotypes (Savarit and Ferveur 2002a). Unsaturated compounds are graphed as black bars; saturated compounds as gray bars.

stimulate male courtship, but are not necessary for courtship and mating (Antony et al. 1985; Ferveur and Sureau 1996; Savarit et al. 1999). Another aphrodisiac hydrocarbon is 7-pentacosene (7$\mathrm{P}$, bar \#10), which is expressed at similar levels by CS males and females (Antony and Jallon 1982; Antony et al. 1985; Ferveur and Sureau 1996; Savarit and Ferveur 2002a). The most effective known inhibitor of male courtship is 7-tricosene (7-T, bar \#4), a 23C monoene (Jallon 1984; Ferveur and Sureau 1996). This antiaphrodisiac is present at low levels in CS female extracts and is the single most abundant $\mathrm{CH}$ in extracts of CS males (Fig. 1A,B, bar \#4). Because analyses of CS virgin and mated female extracts yielded indistinguishable profiles (data not shown), Figure 1A also represents the $\mathrm{CH}$ profile experienced by males during training with mated females. Experimental targets included males and females of various genotypes that expressed different combinations of the compounds in CS female extracts; their $\mathrm{CH}$ profiles (Fig. 1C-H) are described below in the contexts of the corresponding behavioral data.

Initial results confirmed that $1 \mathrm{~h}$ of training with CS mated females results in significant suppression of male courtship toward CS virgin female targets, in tests at both $5 \mathrm{~min}$ and $60 \mathrm{~min}$ after training (Fig. 2A). The role of the females' $\mathrm{CH}$ profile in eliciting the males' learned responses was tested with hs-tra virgin females, which are severely depleted of all CHs (Fig. 1C). Naive males courted $h s$-tra females actively, confirming that the CHs expressed by normal virgins are not required to elicit male courtship (Savarit et al. 1999). Trained males tested with hs-tra virgins displayed no significant suppression of courtship when tested either $5 \mathrm{~min}$ or $60 \mathrm{~min}$ after mated-female training (Fig. 2B). Since male courtship of these $\mathrm{CH}$-depleted virgins is not affected by experience with mated females, the results suggest that the conditioned suppression of courtship observed in tests with normal virgins (Fig. 2A) depends on some component(s) of the normal female $\mathrm{CH}$ profile.

This interpretation was tested with courtship targets bearing a variety of distinctive $\mathrm{CH}$ profiles. These included male flies expressing characteristically female profiles (Fig. 1D,E), virgin females selectively depleted for unsaturated CHs (Fig. 1G), and males expressing subsets of the female $\mathrm{CH}$ profile (Fig. $1 \mathrm{~F}, \mathrm{H}$ ). For example, males heterozygous for certain enhancer-trap pGAL4 inserts and UAS-tra express female $\mathrm{CH}$ profiles (Fig. 1D,E) but retain male morphology (Ferveur et al. 1997; Savarit and Ferveur 2002a). When these GAL4-tra males were used as test targets, they elicited active courtship from naive CS male subjects, while trained males courted them significantly less actively both $5 \mathrm{~min}$ and 60 min after training (Fig. 2D,E). These results show that a female $\mathrm{CH}$ profile on a decapitated male target is sufficient to elicit active courtship from naive males and is a less effective aphrodisiac cue after subjects have been trained with mated females. They further support the hypothesis that component(s) of the hydrocarbon blend, and not female morphological features, function as a conditioned stimulus.

Three additional target genotypes allowed for independent manipulations of different components of the female $\mathrm{CH}$ profile. Males of the wild Tai2 strain naturally express very high levels of monounsaturated pentacosenes 9-P and 7-P (bars \#9 and 10, respectively, in Fig. 1F), but no detectable 7,11-dienes, the aphrodisiacs found specifically on CS females. Because 7-P can also act as an aphrodisiac for CS males (Antony et al. 1985; Sureau and Ferveur 1999), naive subjects court actively in the presence of decapitated Tai 2 males (Fig. 2F). If 7,11-dienes were essential components of the conditioned stimulus, then trained males would be expected to court Tai 2 targets as actively as naives. In fact, trained subjects displayed very strong suppression of court- 
Pheromones in Drosophila courtship conditioning
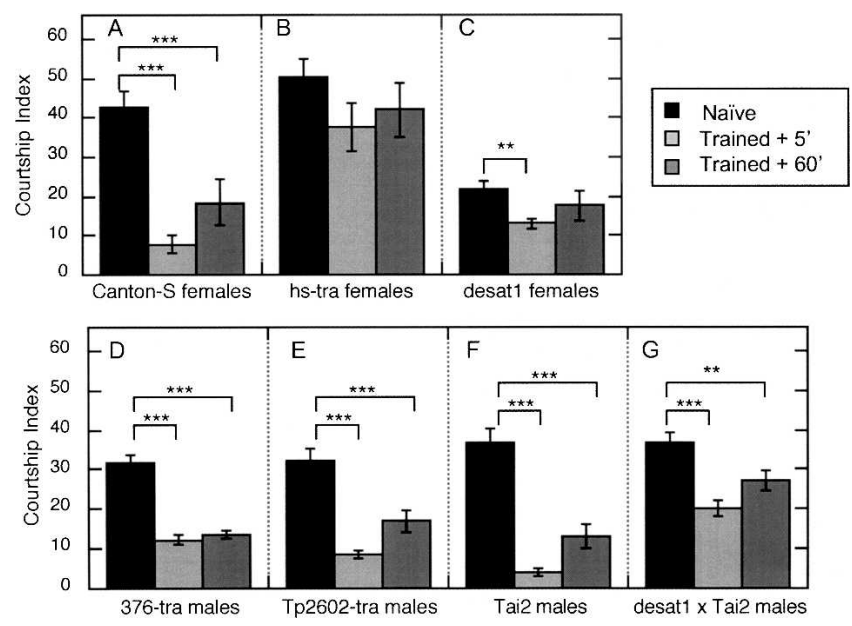

Figure 2. The effects of training with mated females on subsequent courtship of Canton-S males toward different targets. The mean Courtship Indexes (Cls) are plotted ( \pm SEM) for groups of Naive (black bars, $N=38-54$ per group) and Trained (gray bars, $N=19-37$ per group) male subjects tested with decapitated targets of the indicated genotypes, either 5 min (lighter gray bars) or 60 min (dark gray bars) after training. The test targets were $(A)$ Canton-S virgin females, $(B)$ hs-tra virgin females, $(C)$ desat ${ }^{1573}$ virgin females, $(D)$ 376-GAL4/+; UAS-tra/+ males, (E) Tp2602-GAL4/+; UAS-tra/+ males, $(F)$ Tai2 males, and (G) desat1 $1573 \times$ Tai2 males. Differences between the Cls of Trained groups and Naive controls with each target were evaluated by ANOVA; ${ }^{(*)} P<0.01 ;(* *) P<0.001$.

ship in tests with Tai 2 male targets, both 5 min and 60 min after training (Fig. 2F). This result indicates that 7,11-dienes are not essential components of the conditioned stimulus, and that features of the $\mathrm{CH}$ profile expressed by Tai 2 males are sufficient to elicit the effects of mated-female training.

The final two variants on the $\mathrm{CH}$ profile were flies homozygous for desat $1^{1573}$, a pGAL4 insert in the desaturase 1 gene on Chromosome III (Marcillac et al. 2005). Virgin females of this strain are severely (but not entirely) depleted of 7,11-dienes and are highly enriched for saturated CHs, relative to CS virgins (Fig. $1 G)$. Males of the desat $1^{1573} \times$ Tai2 genotype are homozygous for the mutation in a Tai2 genetic background, resulting in no detectable 7,11-dienes and much lower levels of pentacosenes (9-P and 7-P) than wild-type Tai2 males (Fig. 1H). Similar results were obtained in behavioral tests with both of these desat $1^{1573}$ mutant genotypes (Fig. 2C,G): trained males displayed significant suppression of courtship relative to naive controls when tested 5 min after training, and much weaker effects of training after $60 \mathrm{~min}$. At both time points, the effects of training elicited in tests with these desat 1 mutants were far less robust than the learned responses observed in tests with their respective wildtype controls (compare desat1 females to CS females, and desat $1 \times$ Tai 2 males to Tai 2 males in Fig. 2 ). These results indicate that neither high levels of saturated $\mathrm{CHs}$ nor the very low levels of unsaturated $\mathrm{CHs}$ in these mutants are sufficient to elicit strong and sustained memory of courtship conditioning. Also, because memory was actually weaker in tests with desat $1^{1573} \mathrm{fe}-$ males, which express small amounts of 7,11-dienes, and was relatively stronger in tests with the diene-free desat $1^{1573} \times$ Tai2 males (Fig. 2C,G), the results provide further evidence against a role for 7,11-dienes as components of a conditioned stimulus.

These differential effects of training on the Courtship Index (CI) with targets bearing different $\mathrm{CH}$ profiles were reproduced in a second series of experiments, in which the specific types of courtship behaviors performed also were analyzed. For example, while $30 \%-35 \%$ of naive males attempted to copulate with decapitated CS virgin females (Fig. 3A) and with GAL4-tra males bearing a similar $\mathrm{CH}$ profile (Fig. 3D), fewer than 5\% of trained males attempted to copulate with these targets. However, the frequency of copulation attempts with different targets was not always perfectly correlated with the mean CI. Only a few males in either naive or trained groups attempted to copulate with the $\mathrm{CH}$-depleted females of hs-tra and desat1 genotypes (Fig. 3B,C). Also, training did not significantly affect the fraction of males attempting to copulate with either Tai 2 male or desat $1 \times$ Tai2 male targets (Fig. 3E,F). This analysis indicated that mated-female training decreased the fraction of males attempting to copulate with targets expressing a typically female $\mathrm{CH}$ profile.

We next considered whether the learned responses of trained males were significantly correlated with the levels of specific unsaturated hydrocarbons expressed by the test targets. Learning Indexes (LIs) were calculated for males tested with each target genotype, as the percent change in mean CI between trained and naive groups (see Materials and Methods). Correlations between these LI values and the levels of 16 unsaturated compounds in cuticular extracts of target genotypes were identified by simple regression analyses (Table 1). LIs for groups tested 5 min after training (LI5') with nine different targets were most strongly correlated with levels of 9-pentacosene (9-P) (Fig. 4A; Table 1), and were weakly correlated with two other compounds: the anti-aphrodisiac 7-tricosene (7-T) and the barely detectable levels of 5-heptacosene (5-H) (Fig. 4B,C; Table 1). LI5' values were not significantly correlated with the levels of the known aphrodisiacs, 7-P, 7,11-HD, or 7,11-ND (Fig. 4D,E,F; Table 1). The results show that males tested 5 min after training with mated females are more likely to display conditioned courtship suppression toward targets with higher levels of 9-P. This finding was reinforced by results of a similar analysis based on a less extensive data set of males tested $60 \mathrm{~min}$ after training with seven different
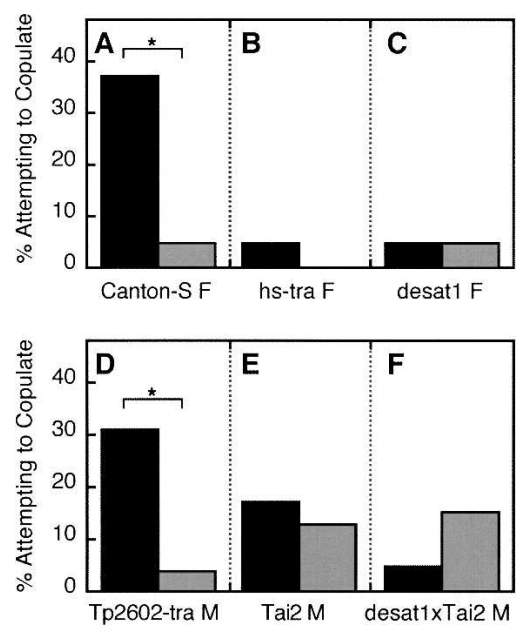

Figure 3. The effects of training with mated females on males' attempts to copulate with different targets during 10-min courtship assays performed $5 \mathrm{~min}$ after training. The percentage of males that attempted to copulate with decapitated targets of the indicated genotypes is plotted for groups of Naive (black bars, $N=19-26$ per group) and Trained (gray bars, $N=20-26$ per group) male subjects. Fewer Trained males than Naives attempted to copulate with $(A)$ Canton-S virgin females $\left(\chi^{2}=6.059, \mathrm{df}=1,\left[^{*}\right] P<0.02\right)$ and $(D)$ Tp2602-tra males $\left(\chi^{2}=6.584\right.$, $\left.\left.\mathrm{df}=1,{ }^{*}\right] P<0.02\right)$, but the frequencies of copulation attempts with other targets were independent of subjects' training status (by $\chi^{2}$ tests of association). These targets were $(B)$ hs-tra virgin females, ( $C$ ) desat 1 virgin females, $(E)$ Tai2 males, and $(F)$ desat $1 \times$ Tai2 males. 

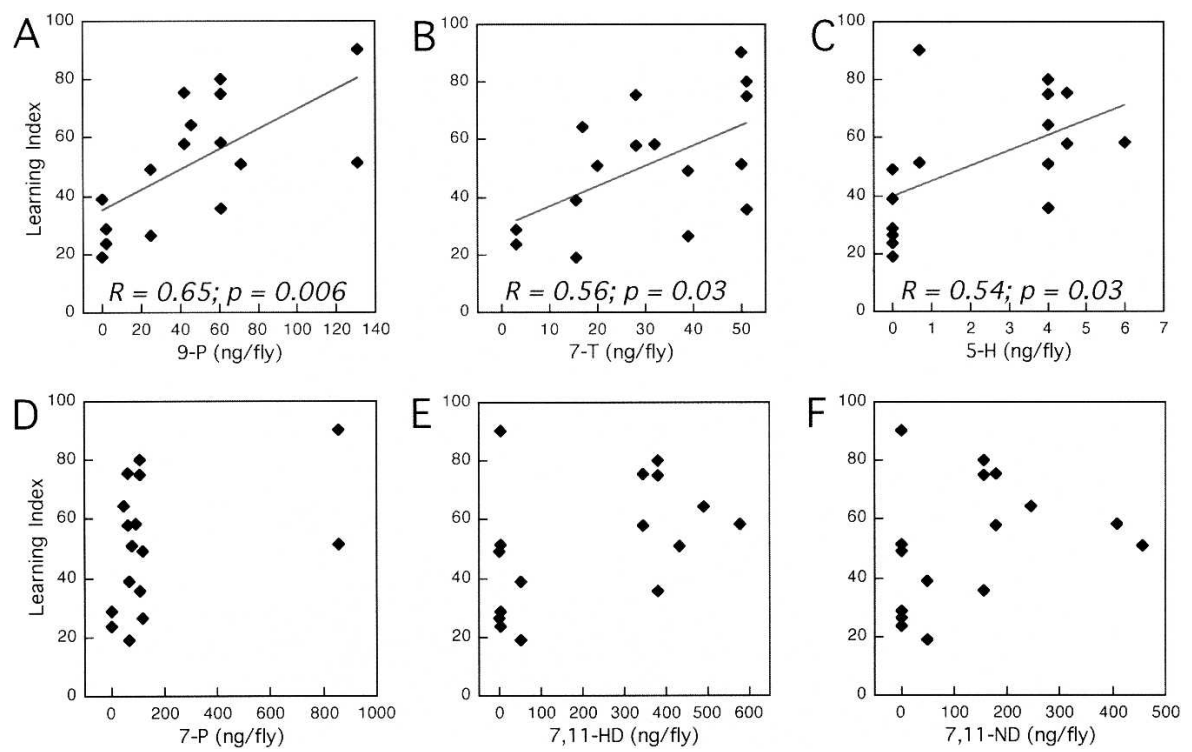

Figure 4. Plots of Learning Index (LI) versus the levels of specific $\mathrm{CHs}$ in different target flies used to assay the effects of courtship conditioning. LI values were calculated as the percent reduction in the mean $\mathrm{Cl}$ of trained males, tested $5 \mathrm{~min}$ after training, relative to naive controls, in a total of 16 experiments involving nine different target genotypes. These $\mathrm{LI}$ values are plotted versus the levels (nanograms/fly) of six individual unsaturated hydrocarbons in cuticular extracts of the target genotypes. $\mathrm{CH}$ profiles for seven of these targets are shown in Figure $1 \mathrm{~A}, \mathrm{C}-\mathrm{H}$; each of these was used in two experiments. Two additional $p G A L 4 /+$; UAS-tra/+ genotypes were used as targets in one experiment; these were Tp3502(GAL4)-tra males and Tp4778(GAL4)-tra females, which both had CH profiles similar to Canton-S females (Savarit and Ferveur 2002a). (A) LI versus 9-P, $(B)$ LI versus 7-T, $(C)$ LI versus 5-H, $(D)$ LI versus 7-P, $(E)$ LI versus 7,11-HD, and $(F)$ LI versus 7,11-ND. Linear fits and correlation coefficients $(R)$ are indicated only for the three $\mathrm{CHs}$ for which the simple regressions yielded $P<0.05$. Also see Table 1.

target genotypes, where 9-P again emerged as the compound with which LI60' values were most strongly correlated (Table 1).

\section{The effects of 9-pentacosene on courtship of naive}

\section{and trained males}

Results of this correlation analysis suggest that courtship conditioning might alter males' responses to 9-P, such that it either acquires anti-aphrodisiac properties or becomes less potent in its aphrodisiac effects as a consequence of training. We tested this directly by adding pure, synthetic (Z)-9-pentacosene (kindly provided by L.M. Hanks [University of Illinois] and J.G. Millar [University of California-Riverside]) to test targets that naturally expressed no detectable levels of this $\mathrm{CH}$. These targets were hs-tra virgin females, desat1 virgin females (see $\mathrm{CH}$ profiles in Fig. 1), and immature Canton-S males. The cuticular hydrocarbons of immature Canton-S males (3-6 h after eclosion) are mainly 27C to 35C, and do not include detectable levels of 9-P (Pechine et al. 1988). 9-P was added in $20 \mu \mathrm{L}$ of hexane to a filter paper disk in each test chamber, and the hexane was allowed to evaporate before the flies were added. Control tests were performed on filter disks treated with $20 \mu \mathrm{L}$ of hexane alone and confirmed the results of earlier experiments (Fig. 2; Gailey et al. 1984): No significant effects of training were detected in control assays (hexane only) with any of the 9-P-depleted test targets (Figs. 5B,C,D). In parallel tests with CS female targets, the courtship of trained males was strongly suppressed relative to naives (Fig. 5A). This effect of training was similar both in control tests and when $2 \mu \mathrm{g}$ of 9-P (about 30 times the average amount of 9-P in cuticular extracts of CS virgins) was added to the filter disks (Fig. 5A), indicating that these treatments did not interfere with the expression of the conditioned response. Addition of 9-P (either 0.2 $\mu \mathrm{g}$ or $2 \mu \mathrm{g}$ ) did not affect the CIs of either naive or trained males tested with either $h s$-tra virgins or immature CS male targets (Fig. 5B,D), but 9-P did significantly increase male courtship of desat1 virgins (Fig. 5C). Specifically, mean CIs with desat 1 virgins were higher with the higher dose of 9-P than in either hexane controls or the lower 9-P dose. The data in Figure 5C suggest that there was a greater tendency for naive males than for trained males to increase their courtship of desat1 virgins at the higher dose of 9-P, but the interaction between training condition and 9-P dose was not significant (by two-way ANOVA, $P=0.13)$. Closer examination of these results revealed dose-dependent effects of 9-P on the Learning Index expressed in tests with $\mathrm{CH}$-depleted targets (Table 2). Clear increases in LI5' were found both between control and $0.2 \mu \mathrm{g}$ of 9-P, and between $0.2 \mu \mathrm{g}$ and $2 \mu \mathrm{g}$ of 9-P, when effects of training were assayed with $h s$-tra females, desat 1 females, and immature CS males. No such effect was found in tests with CS females.

Even more dramatic effects of 9-P emerged when the specific behavioral stages of courtship were analyzed. Results of earlier experiments (see Fig. 3 above) showed that training dramatically decreased the fraction of males attempting to copulate with test targets expressing female $\mathrm{CH}$ profiles, while very few males in either trained or naive groups attempted to copulate with the $\mathrm{CH}$-depleted $h s$-tra or desat 1 mutant females. The essential features of these results were confirmed in control conditions (hexane only) of the 9-P experiments: naive males attempted to copulate with CS female targets at a much greater frequency than trained males (Fig. 6A), but similar numbers of trained and naive males attempted to copulate with the $\mathrm{CH}$-depleted targets in control tests (Fig. 6B,C,D). The effects of adding 9-P were to increase the fraction of males attempting to copulate with CS virgin females (Fig. 6A), hs-tra virgins (Fig. 6B), and desat1 virgins (Fig. 6C), but a slight increase in copulation attempts with immature male targets was not significant (Fig. 6D). Effects of training, as indicated by a higher frequency of copulation attempts by naive males than by trained males, were increased between control and 0.2- $\mu$ g-of-9-P conditions with the CH-deficient courtship targets (Fig. 6B,C,D). These effects of training were less evident with the higher 9-P dose, however, because both naive and trained groups increased their copulation attempts between the $0.2-\mu \mathrm{g}$ and $2-\mu \mathrm{g}-9$-P doses. Both naive and trained males also increased their copulation attempts with CS females between control and 2- $\mu$ g-9-P conditions. Overall, the results indicate that 9-P increased copulation attempts by CS males; the lower dose of 9-P elicited this effect for naive males, while trained males responded only to the higher dose.

\section{Discussion}

The goal of this study was to identify specific female cues that function as a conditioned stimulus in the courtship conditioning paradigm of $D$. melanogaster, wherein males learn to suppress their courtship toward virgin females as a result of unsuccessful courtship with previously mated females. The results confirm previous suggestions that the conditioned stimulus is a chemo- 
A

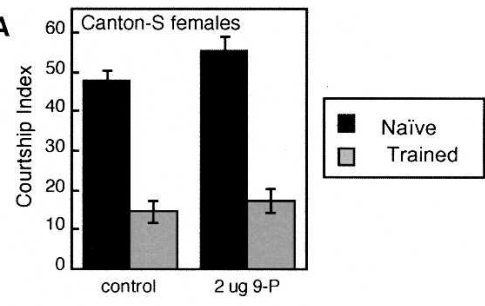

B

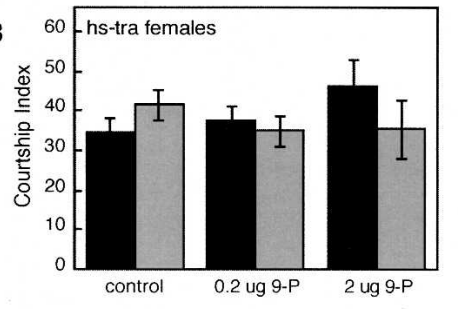

C
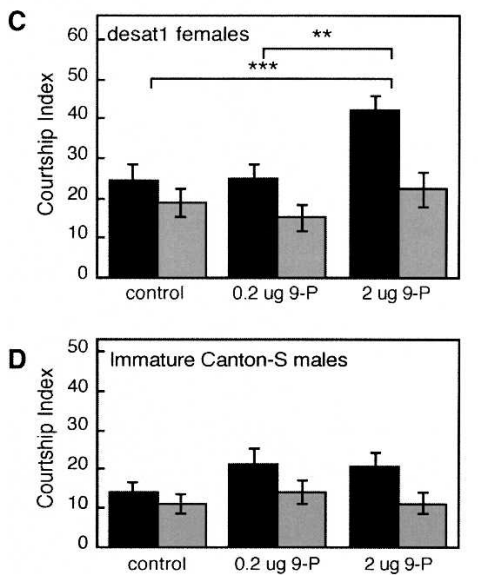

Figure 5. The effects of exogenous 9-pentacosene (9-P) on the Courtship Index ( \pm SEM) of Naive (black bars) and Trained (gray bars, tested 5 min after training) males with $(A)$ Canton-S virgin females $(N=20$ per group), and three additional courtship targets that express no detectable 9-P among their cuticular hydrocarbons: $(B)$ hs-tra virgin females ( $N=11-28$ per group), (C) desat $1^{1573}$ virgin females $(N=20-21$ per group), and $(D)$ immature Canton- $S$ males $(N=20$ per group). Two-way ANOVA indicated a significant effect of 9-P only in tests with desat $1^{1573}$ females $\left(F_{2,117}=7.778, P<0.01\right)$, where Cls with the high dose of 9-P were significantly greater than in the control or low dose conditions ([**] $P<0.01$, $\left.{ }^{* * *}\right] P<0.001$ by Fisher's PLSD). The two-way ANOVA also revealed significant effects of training on $\mathrm{Cls}$ with Canton-S females $\left(F_{1,89}=143.2, P<0.0001\right)$, with desat $1^{1573}$ females $\left(F_{1,117}=16.22\right.$, $P=0.0001)$, and with immature Canton-S males $\left(F_{1,114}=6.391\right.$, $P<0.05)$. No significant interactions were found between training and 9-P dose.

sensory cue (Tompkins et al. 1983; Gailey et al. 1984; Ejima et al. 2005), and provide direct evidence that a minor cuticular hydrocarbon functions as a significant component of this conditioned stimulus. Based on the reasoning that trained males would exhibit a learned response only in courtship tests with targets that expressed a conditioned stimulus, flies with different combinations of putative female aphrodisiac cues were used as test targets, and assessed for their ability to elicit a learned response from CS males. After $1 \mathrm{~h}$ of experience courting a previously mated CS female, the effects of this experience were measured in courtship assays with different test targets. Differences in the extent and duration of conditioned courtship suppression in tests with different targets were attributed to differences in the testing conditions, that is, in the combinations of aphrodisiac and antiaphrodisiac cues expressed by the test targets.

Target behavior was eliminated as a source of the observed differences in male courtship suppression because the targets were immobilized by decapitation. Nor did the presence of female abdominal anatomy correlate with a target's ability to elicit a learned response: Targets that were anatomically female and male elicited strong learned responses, while the target genotypes that elicited the weakest learned responses (desat $1^{1573}$ virgins and $h s$-tra virgins) were anatomically female (Fig. 2). Therefore, the abdominal anatomy of a courtship target is not an essential element of the conditioned stimulus. This conclusion also is supported by the fact that visual stimuli are not required for courtship conditioning (Tompkins et al. 1983; Joiner and Griffith 1997).

The courtship activity (mean CI) of naive males was not well correlated with the hydrocarbon profiles of the different test targets, confirming the results of prior studies showing that female $\mathrm{CHs}$ are sufficient, but not necessary, to stimulate naive male courtship (Antony et al. 1985; Savarit et al. 1999). In particular, naive males courted hydrocarbon-depleted $h s$-tra virgins and males depleted of unsaturated CHs as actively as they courted targets with typically female $\mathrm{CH}$ profiles. This is consistent with the view that naive males respond to a combination of aphrodisiac and anti-aphrodisiac pheromones on a decapitated target fly. In contrast, the conditioned suppression of courtship resulting from experience with mated females was closely correlated with the hydrocarbon profiles of the test targets. Results of testing with hydrocarbon-depleted female targets indicated that CHs are essential stimuli for the conditioned suppression of courtship after experience with mated females (Fig. 2B). Tests with feminized males (pGAL4/+; UAS-tra/+) that expressed typically female $\mathrm{CH}$ profiles revealed that this profile is sufficient both to stimulate naive male courtship and to elicit conditioned suppression of courtship in trained male subjects (Fig. 2D,E). Together, these results strongly support the hypothesis that components of the female $\mathrm{CH}$ profile function as the conditioned stimulus in courtship conditioning.

This raises the question of whether specific compounds or the overall female profile are responsible for the learned response. The data indicate that a target's ability to elicit a learned response was most strongly correlated with the levels of a single monoene, 9-pentacosene (9-P). Specifically, LIs based on tests either $5 \mathrm{~min}$ or $60 \mathrm{~min}$ after training were significantly correlated with target levels of a single unsaturated hydrocarbon, 9-P (Table 1). Surprisingly, the LIs were not significantly correlated with levels of any principal compound previously known to possess aphrodisiac properties, that is, the 7,11-dienes or 7-P (Fig. 4D,E,F).

Interpretation of this result requires consideration of previous evidence concerning the aphrodisiac properties of individual CHs. Two strategies have been used to assess the aphrodisiac and anti-aphrodisiac potencies of individual compounds in the cuticular profile. By applying synthetic compounds to $\mathrm{CH}$-stripped dummy targets, Jallon and coworkers found 7,11-HD to be the most bioactive, stimulating aggressive male courtship with a threshold of $\sim 100 \mathrm{ng} /$ fly (Antony and Jallon 1982; Antony et al.

Table 2. Effects of 9-P on Learning Indexes (LI5') obtained in tests with different courtship targets

\begin{tabular}{lrcc}
\hline Courtship target & Control & $\mathbf{0 . 2} \boldsymbol{\mu g}$ 9-P & 2 $\boldsymbol{\mu g}$ 9-P \\
\hline Canton-S females & 70 & & 69 \\
hs-tra females & -20 & 3.4 & 23 \\
desat1 females & 23 & 40 & 47 \\
Immature Canton-S males & 22 & 34 & 45 \\
\hline
\end{tabular}

See Figure 5. Greater LI5'-values were obtained with increasing doses of 9-P in tests with $\mathrm{CH}$-depleted targets, but not with Canton-S females. 
A

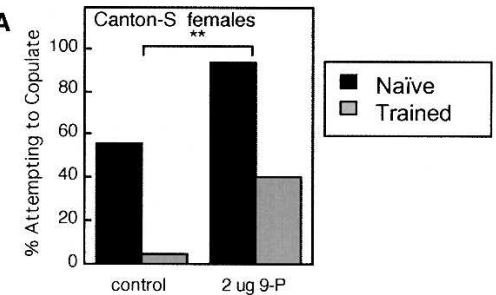

B

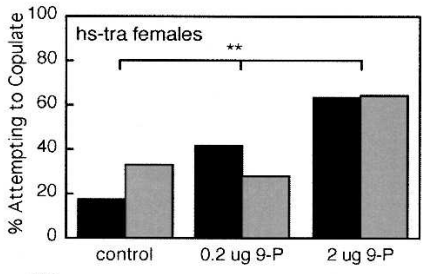

C

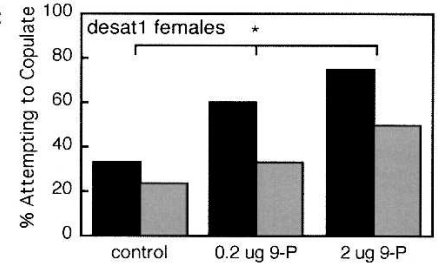

D

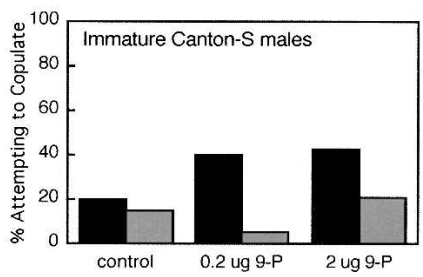

Figure 6. The effects of exogenous 9-P on the frequency of copulation attempts of Naive (black bars) and Trained (gray bars, tested 5 min after training) males with different courtship targets, from the same experiment for which $\mathrm{Cls}$ are plotted in Figure 5. Possible effects of training and of 9-P were evaluated independently by $\chi^{2}$ tests of association. The effect of 9-P (with Naive and Trained groups combined; thus brackets are positioned above each pair of bars) was to significantly increase the percent of males attempting to copulate with $(A)$ Canton-S females $\left(\chi^{2}=6.988\right.$ $\left.\mathrm{df}=1,{ }^{\left.{ }^{* *}\right]} P<0.01\right),(B)$ hs-tra females $\left.\left(\chi^{2}=11.01, \mathrm{df}=2,{ }^{* *}\right] P<0.01\right)$, and $(C)$ desat $1^{1573}$ virgin females $\left(\chi^{2}=8.200, \mathrm{df}=2,\left[^{*}\right] P<0.02\right)$, but not with $(D)$ immature Canton-S males $\left(\chi^{2}=2.800, \mathrm{df}=2, P=0.2\right)$. Similarly, effects of training were assessed across all 9-P doses; these tests revealed a significant association between training status and attempts to copulate with $(A)$ Canton-S females $\left(\chi^{2}=18.26\right.$, df $\left.=1, P<0.0001\right)$, (C) desat $1^{1573}$ females $\left(\chi^{2}=5.927, \mathrm{df}=1, P<0.02\right)$, and $(D)$ immature Canton-S males $\left(\chi^{2}=7.420, \mathrm{df}=1, P<0.01\right)$, but not with $(B)$ hs-tra females $\left(\chi^{2}=0.001\right.$, df $\left.=1, P=0.97\right)$.

1985). This was confirmed by Ferveur and Sureau (1996), using genetic manipulations to produce live targets with distinctive $\mathrm{CH}$ profiles, who also found that even low levels of 7,11-ND (10-15 ng) could strongly potentiate the aphrodisiac effects of 7,11-HD. Both strategies produced evidence for the anti-aphrodisiac properties of 7-T and the aphrodisiac effects of 7-P (Antony et al. 1985; Sureau and Ferveur 1999). Prior evidence regarding the putative aphrodisiac properties of 9-P is limited to a positive correlation with the frequency of copulation attempts (Ferveur and Sureau 1996). When applied to hydrocarbon-stripped targets, 9-P failed to stimulate male courtship (Antony et al. 1985).

In the present study, one clear effect of adding exogenous, pure (Z)-9-pentacosene to courtship chambers was to increase the fraction of CS males that attempted to copulate with CS virgin females, and with $\mathrm{CH}$-depleted virgins of the hs-tra and desat1 genotypes (Fig. 6). This result strongly supports the hypothesis that the main aphrodisiac effect of 9-P is to increase the likelihood of copulation attempts (Ferveur and Sureau 1996; Ferveur 1997). Interestingly, 9-P did not increase the mean CI in most of these test groups: Only naive males tested with desat 1 females had a higher mean CI in the presence of 9-P (Fig. 5C). This selective effect on the mean CI of naive males with desat 1 females, but not trained males, as well as the dose-dependent effects of 9-P on the Learning Index (Table 2), clearly establish a role for 9-P as a component of the conditioned stimulus. With most targets, however, 9-P did not stimulate overall courtship activity; rather, it increased the probability for courting males to attempt copulations. Moreover, because this effect was elicited by the lower dose of 9-P for naive males, and only at the higher dose for trained males, it suggests that a specific effect of training with mated females is to reduce the responsiveness of trained males to the aphrodisiac effects of this pheromone.

These results indicate a dual role of 9-P: (1) increasing the probability that courting males will attempt to copulate, and (2) contributing to the difference between naive and trained males in overall courtship. Yet, depending on the target genotype, different doses of 9-P induced different effects on the global amount of courtship (CI) and on the frequency of copulation attempts. For example, 9-P only increased the CI of naive males when present at $2 \mu \mathrm{g}$ on desat 1 females, but both $0.2-\mu \mathrm{g}$ and $2-\mu \mathrm{g}$ doses tended to increase the probability to attempt copulation when combined with any of the four targets. This difference can be explained because the duration of a copulation attempt is very brief compared to the wing vibration and licking behaviors that make up most of the CI measure (Ferveur and Sureau 1996). The selective effect of 9-P on CI with desat1 females suggests that its effect could be potentiated by other $\mathrm{CHs}$ that are present in these targets but absent in $h s$-tra females and immature males. At least two relatively abundant CHs fit this criterion: $n$-tricosane (\#6; Table 1) and 2-methyl-tetracosane (\#8). On the other hand, it is likely that 9-P had minimal effects on CIs with CS females because all the $\mathrm{CHs}$ necessary to induce high levels of courtship were already present, and the exogenous 9-P did not increase the overall sex appeal of these targets. It was also with the three $\mathrm{CH}$-depleted genotypes, but not with CS females, that dosedependent effects of 9-P were found on LI5', a measure of the relative suppression of overall courtship in trained males, whereas $0.2 \mu \mathrm{g}$ of 9-P was sufficient to elicit the maximum effect of training on the probability to attempt copulation. It is worth noting that the 0.2- $\mu$ g dose of 9-P (applied to a filter paper under the target fly) is very similar to the biological dose found on CS females (50-70 ng). Thus, the results demonstrate that CS males can detect and respond to physiologically relevant levels of this pheromone.

It is likely that 9-P is one component of a combination of $\mathrm{CHs}$ that function as the conditioned stimulus. This is supported by the fact that 9-P was not sufficient to elicit effects of training on courtship of $\mathrm{CH}$-depleted $h s$-tra females (Figs. 5B, 6B). It is also supported by the modest, albeit significant, correlation between the LIs and 9-P levels in different test targets (Fig. 4A). When possible synergistic effects of other unsaturated $\mathrm{CHs}$ with 9-P were examined by running multiple regressions, there were no cases in which the levels of 9-P plus another $\mathrm{CH}$ predicted the LI-values significantly better than did 9-P alone. Thus, the identities of the putative interacting components of the conditioned stimulus remain to be determined.

The surprising result of this study is that this particular compound emerged as a conditioned stimulus. The levels of 7,11dienes in a courtship target were not correlated with the expression of the males' learned response (Fig. 4E,F), indicating that conditioning does not dramatically alter the potency of these highly abundant, female-specific pheromones. In contrast, 9-P is 
a relatively minor component of the Canton-S $\mathrm{CH}$ profile, and is expressed by both mature males and females.

Having identified a role for 9-P as a chemosensory stimulus that is particularly susceptible to conditioning during courtship of mated females, the results may shed new light on the question of whether courtship conditioning ability is relevant to the behavior of males in the wild. While it is likely to be maladaptive for males to suppress their courtship toward conspecific virgin females, Gailey and colleagues (1984) proposed that courtship toward mated females might be decreased to a greater degree, thus improving male selectivity for virgins. As 9-P is not very specific (it is found in cuticular extracts of both males and females of several Drosophila species) (Jallon and David 1987; Howard et al. 2003), it is possible that experience-dependent modification of the responsiveness to this widely expressed hydrocarbon might provide a male with the ability to learn to discriminate appropriate from inappropriate courtship targets. For example, females of Drosophila affinis were reported to express much higher levels of 9-P than D. melanogaster females (Jackson 1996; Ferveur 2005). These two species are sympatric in some areas of North America, and they have been observed courting in interspecific groups gathered on food sources (McRobert and Tompkins 1986). These observations suggest that the ability of CS males to learn to be less responsive to 9-P might allow them to learn to avoid courting $D$. affinis females. In an environment where misdirected courtship of heterospecific females is likely to occur, there would presumably be some advantage for males that could learn to become less sensitive to particular pheromones that are more abundant in heterospecific females than in conspecifics.

D. melanogaster males also court immature males and females, which express distinctly different blends of $\mathrm{CHs}$ than mature flies (Pechine et al. 1988; Ejima et al. 2005). Experience courting immature males results in habituation to the aphrodisiac effects of specific 31C monoenes in the immature male $\mathrm{CH}$ blend (Gailey et al. 1982; Vaias et al. 1993). The $\mathrm{CH}$ profiles of immature males and females are similar to each other in that they are comprised mostly of longer 27C-37C compounds and contain only trace amounts of compounds smaller than $27 \mathrm{C}$ (Pechine et al. 1988; Ejima et al. 2005). It is difficult to predict how experience courting a mated female, and the corresponding decrease in aphrodisiac potency of 9-P, might affect a male's subsequent courtship of immature flies with little or no 9-P and an abundance of other CHs with unknown behavioral effects. This difficulty is compounded by the possibility that some longer-chain $\mathrm{CHs}$ of immature flies may be detected and processed through some of the same sensory pathways as mature $\mathrm{CH}$ pheromones, a possibility that is reinforced by recent evidence that many gustatory receptor neurons express combinations of receptors (Amrein 2004; Thorne et al. 2004). At present, therefore, it is not possible to interpret the effects of mated-female training on male courtship of immature males and females (Ejima et al. 2005) in terms of specific modifications of responses to specific CHs.

The present results suggest an additional level of complexity in the functional organization of the neural systems that control and modulate male courtship. Some CHs (7,11-dienes and 7-P) stimulate naive males to court actively; and others (7-T) inhibit naive courtship, suggesting that there are at least two distinct gustatory pathways by which contact pheromones influence courtship control centers (Bray and Amrein 2003; Stockinger et al. 2005). Males repeatedly sample all of these pheromones during training with mated females, yet the experience-dependent changes in subsequent courtship behavior are strongly correlated only with targets' levels of 9-P. This suggests that the sensorimotor circuits by which 7,11-dienes and 7-P stimulate courtship may be less modifiable than pathways activated by 9-P. It follows from this hypothesis that these pheromones may be detected and processed by distinct neural pathways, a prediction that can be tested by manipulating the genes and cells involved in pheromone detection (Xu et al. 2002; Bray and Amrein 2003; Stockinger et al. 2005).

In this study, we have provided direct support for the hypothesis that the conditioned stimulus in courtship conditioning of $D$. melanogaster males is a chemical, rather than behavioral or anatomical, cue provided by the female (Tompkins et al. 1983; Gailey et al. 1984). Our results indicate that components of the female cuticular hydrocarbon profile function as the conditioned stimulus in courtship conditioning. In particular, the most relevant stimulus is 9-pentacosene, an unsaturated hydrocarbon found on the cuticle of both males and females. Together with previous evidence concerning the relative potencies of various cuticular substances at eliciting male courtship, the present results suggest that naive males respond to a combination of aphrodisiacs and anti-aphrodisiacs on a target fly, while conditioned males are less responsive than naives to the aphrodisiac effects of 9-P. Identifying the sensory pathways responsible for detection of this chemical conditioned stimulus will allow for a more definitive analysis of the neural mechanisms responsible for this form of associative learning.

\section{Materials and Methods}

\section{Drosophila strains}

All flies were reared at $25^{\circ} \mathrm{C}$ on cornmeal-sucrose-yeast-agar medium, and were exposed to a 12-h light/12-h dark cycle. Wildtype Canton-S (CS) and Tai2 strains and transgenic strains for pheromonal manipulations (UAS-tra $20 J 7$ and pGAL4 enhancer trap strains) were from lines maintained in the Ferveur lab. The hsp70-GAL4.PB strain was obtained from the Bloomington Stock Center (1799).

Hydrocarbon-depleted $h s$-tra females were the progeny of crosses between UAS-tra $20 J 7$ males and hsp70-GAL4.PB females: hsp70-GAL4.PB/+; UAS-tra/+ virgin females were subjected to a 1-h heat shock $\left(37^{\circ} \mathrm{C}\right) 5-6 \mathrm{~h}$ after eclosion (Savarit et al. 1999; Savarit and Ferveur 2002b). It is thought that early toxic effects of hsp70-GAL4 induction in cells responsible for $\mathrm{CH}$ synthesis (i.e., oenocytes) may explain the near absence of detectable $\mathrm{CHs}$ in these females 4-5 d later (Savarit and Ferveur 2002b), when they were used as test targets in courtship assays. Male targets with female $\mathrm{CH}$ profiles were the progeny of crossing UAS-tra $20 J 7$ males with females of different enhancer trap GAL4 strains (376GAL4, Tp2602-GAL4, and Tp3502-GAL4), which drive expression in oenocytes and thereby feminize the profile of $\mathrm{CHs}$ in the respective GAL4/+; UAS-tra/+ male progeny (Savarit and Ferveur 2002a). In desat $1^{1573}$ mutant females, a pGAL4 insertion in the desaturase 1 gene on Chromosome III results in a dramatic reduction of unsaturated $\mathrm{CHs}$ and increase in saturated compounds (Marcillac et al. 2005). Males of the desat $1^{1573} \times$ Tai2 genotype were from a stock in which Chromosome II of the Tai2 strain (that changes the 7-T: 7-P ratio) was combined with Chromosome III of the desat $1^{1573}$ strain.

\section{Hydrocarbon extraction and gas chromatography}

Cuticular hydrocarbons (CHs) of individual flies were extracted by a standard hexane wash, and levels of identified hydrocarbons were measured by gas chromatography, as previously described (Ferveur 1991; Savarit and Ferveur 2002a). Peaks were identified based on time of elution, and amounts were calculated based on comparison to internal standards. Data from 6-12 individual flies were averaged; there was little variation in $\mathrm{CH}$ levels among flies of the same genotype.

\section{Behavioral assays}

All flies were collected under $\mathrm{CO}_{2}$ anesthesia within $5 \mathrm{~h}$ of eclosion. CS male subjects were aged for $4-5 \mathrm{~d}$ in individual small 
glass tubes containing normal cornmeal medium. They were transferred to fresh individual tubes $15-24 \mathrm{~h}$ prior to experiments. CS females used for training were aged 4-6 d in groups of $5-15$, then paired individually with CS males for pre-mating. Females observed to mate were stored in groups of five until being used to train subject males the following day.

Training and testing took place during the first $5 \mathrm{~h}$ after lights-on, at ambient temperature $\left(23^{\circ}-26^{\circ} \mathrm{C}\right)$ with fluorescent room lighting. Courtship chambers were created by placing a watch glass $\left(3.5 \mathrm{~cm}^{2}\right)$ on a glass plate (Savarit et al. 1999). For the training phase, healthy CS males were placed in individual chambers; 5 min later, mated females were randomly added to about half the chambers. Both sham-trained males (Naive) and males with mated females (Trained) were left in the chambers for 60 min, then the male subjects were aspirated to clean courtship chambers. After 5 or $60 \mathrm{~min}$, a decapitated target fly was added to assay the male's courtship activity.

Target flies were anesthetized with $\mathrm{CO}_{2}$ and decapitated $\sim 20$ min prior to testing. Only targets that resumed a standing position were used. Once a target was added to a testing chamber, an observer who was blind to the training status of the subject flies recorded the total duration of all courtship activity by each subject male during a 10-min testing period. The latency to initiate courting and the occurrence of specific behaviors (tapping, wing vibration, licking, and attempting copulation) were also recorded.

In some experiments, synthetic (Z)-9-pentacosene (kindly provided by J.G. Millar and L.M. Hanks) was added to filter paper disks (Whatman \#1) in courtship chambers in a 20- $\mu \mathrm{L}$ volume of hexane, and the hexane was allowed to evaporate before the flies were added. Control tests were performed on filter disks treated with $20 \mu \mathrm{L}$ of hexane alone.

\section{Data analyses}

A Courtship Index (CI) was calculated for each male subject by dividing the total time courting by the duration of the testing period $(600 \mathrm{sec})$. After confirming that the CI values were approximately normally distributed within each group, the CIs of naive and trained groups with each type of target were compared by ANOVAs (using StatView 5.0.1). The effects of training and of 9-P dose on the fraction of males in each group that attempted to copulate with a target were evaluated by $\chi^{2}$ tests of association (using JMP 5.0.1).

Learning Indexes (LIs) were calculated to express the effects of training on males tested at $5 \mathrm{~min}$ and $60 \mathrm{~min}$ with each target type.

$$
\text { Learning Index }(\mathrm{LI})=\frac{(\text { mean Naive CI }- \text { mean Trained CI })}{\text { mean Naive CI }} \times 100
$$

Correlations between LI values and the levels of 16 individual hydrocarbons in cuticular extracts of target genotypes were determined by simple regression analyses (using StatView 5.0.1). An adjustment for multiple comparisons was determined by running Monte Carlo simulations, in which the maximum correlation coefficient $\left(R_{\max }\right)$ was determined for 1000 random data sets of $16 Y$-values and 16 different randomly generated $X$-values. The simulation indicated that maximum correlation coefficients greater than $R_{\max }=0.647$ occurred with $P<0.05$.

\section{Acknowledgments}

We gratefully acknowledge the contributions of Claude Everaerts for assistance with the gas chromatography; Jocelyn Millar and Larry Hanks for providing pure, synthetic (Z)-9-pentacosene; Steve Wang for significant help with statistical analyses; and Scott McRobert for thoughtful discussions. This work was supported by NSF Grant \#IBN-9723920, HHMI Undergraduate Science Education Program Grant \#71100505804, and a Lang Faculty Fellowship from Swarthmore College, and by the CNRS and the Burgundy Regional Council.

\section{References}

Ackerman, S.L. and Siegel, R.W. 1986. Chemically reinforced conditioned courtship in Drosophila: Responses of wild-type and the dunce, amnesiac, and don giovanni mutants. J. Neurogenet. 3: 111-123.

Amrein, H. 2004. Pheromone perception and behavior in Drosophila. Curr. Opin. Neurobiol. 14: 435-442.

Antony, C. and Jallon, J.-M. 1982. The chemical basis for sex recognition in Drosophila melanogaster. J. Insect Physiol. 28: 873-880.

Antony, C., Davis, T.L., Carlson, D.A., Pechine, J.-M., and Jallon, J.-M. 1985. Compared behavioral responses of male Drosophila melanogaster (Canton S) to natural and synthetic aphrodisiacs. J. Chem. Ecol. 11: 1617-1629.

Bastock, M. and Manning, A. 1955. The courtship of Drosophila melanogaster. Behaviour 8: 85-111.

Bray, S. and Amrein, H. 2003. A putative Drosophila pheromone receptor expressed in male-specific taste neurons is required for efficient courtship. Neuron 39: 1019-1029.

Cobb, M. and Ferveur, J.-F. 1996. Evolution and genetic control of mate recognition and stimulation in Drosophila. Behav. Proc. 35: 35-54.

Connolly, K. and Cook, R. 1973. Rejection responses by female Drosophila melanogaster: Their ontogeny, causality and effects upon the behaviour of the courting male. Behaviour 44: 142-165.

Davis, R.L. 1996. Physiology and biochemistry of Drosophila learning mutants. Physiol. Rev. 6: 299-317.

Dubnau, J., Chiang, A.-S., and Tully, T. 2003. Neural substrates of memory: From synapse to system. J. Neurobiol. 54: 238-253.

Ejima, A., Smith, B.P.C., Lucas, C., Levine, J.D., and Griffith, L.C. 2005. Sequential learning of pheromonal cues modulates memory consolidation in trainer-specific associative courtship conditioning. Curr. Biol. 15: 194-206.

Ferveur, J.-F. 1991. Genetic control of pheromones in Drosophila simulans. I. Ngbo, a locus on the second chromosome. Genetics 128: 293-301.

. 1997. The pheromonal role of cuticular hydrocarbons in Drosophila melanogaster. BioEssays 19: 353-358.

. 2005. Cuticular hydrocarbons: Evolution and role in the pheromonal communication of Drosophila. Behav. Genet. 35: $279-295$.

Ferveur, J.-F. and Sureau, G. 1996. Simultaneous influence on male courtship of stimulatory and inhibitory pheromones produced by live sex-mosaic Drosophila melanogaster. Proc. R. Soc. Lond. B 263: 967-973.

Ferveur, J.F., Savarit, F., O'Kane, C.J., Sureau, G., Greenspan, R.J., and Jallon, J.M. 1997. Genetic feminization of pheromones and its behavioral consequences in Drosophila males. Science 276: $1555-1558$.

Gailey, D.A., Jackson, F.R., and Seigel, R.W. 1982. Male courtship in Drosophila: The conditioned response to immature males and its genetic control. Genetics 102: 771-782.

. 1984. Conditioning mutations in Drosophila melanogaster affect an experience-dependent behavioral modification in courting males. Genetics 106: 613-623.

Greenspan, R. and Ferveur, J.-F. 2000. Courtship in Drosophila. Annu. Rev. Genet. 34: 205-232.

Hall, J.C. 1994. The mating of a fly. Science 264: 1702-1714.

Howard, R.W., Jackson, L.L., Banse, H., and Blows, M.W. 2003. Cuticular hydrocarbons of Drosophila birchii and D. serrata: Identification and role in mate choice in D. serrata. J. Chem. Ecol. 29: 961-976.

Jackson, L.L. 1996. Involvement of cuticular hydrocarbons in the incomplete behavioral isolation between Drosophila affinis and Drosophila melanogaster. In The $37^{\text {th }}$ Drosophila Conference, San Diego. Abstract, p. 320.

Jallon, J.-M. 1984. A few chemical words exchanged by Drosophila during courtship and mating. Behav. Genet. 14: 441-478.

Jallon, J.-M. and David, J.R. 1987. Variations in cuticular hydrocarbons among the eight species of the Drosophila melanogaster subgroup. Evolution 41: 294-302.

Joiner, M.A. and Griffith, L.C. 1997. CaM kinase II and visual input modulate memory formation in the neuronal circuit controlling courtship conditioning. J. Neurosci. 17: 9384-9391.

Kamyshev, N.G., Iliadi, K.G., and Bragina, J.V. 1999. Drosophila conditioned courtship: Two ways of testing memory. Learn. Mem. 6: $1-20$.

Marcillac, F., Bousquet, F., Alabouvette, J., Savarit, F., and Ferveur, J.-F. 2005. A mutation with major effects on Drosophila melanogaster sex pheromones. Genetics (in press).

McBride, S.M.J., Giuliani, G., Choi, C., Krause, P., Correale, D., Watson, K., Baker, G., and Siwicki, K.K. 1999. Mushroom body ablation impairs short-term memory and long-term memory of courtship conditioning in Drosophila melanogaster. Neuron 24: 967-977.

\section{Learning \& Memory}


McRobert, S.P. and Tompkins, L. 1986. Incomplete behavioral isolation between two distantly related Drosophila species. Evolution 40: $1185-1190$.

Pechine, J.M., Antony, C., and Jallon, J.-M. 1988. Precise characterization of cuticular compounds in young Drosophila by mass spectrophotometry. J. Chem. Ecol. 14: 1071-1085.

Roman, G. and Davis, R.L. 2001. Molecular biology and anatomy of Drosophila olfactory associative learning. BioEssays 23: 571-581.

Savarit, F. and Ferveur, J.-F. 2002a. Genetic study of the production of sexually dimorphic cuticular hydrocarbons in relation with the sex-determination gene transformer in Drosophila melanogaster. Genet. Res. Camb. 79: 23-40.

2002b. Temperature affects the ontogeny of sexually dimorphic cuticular hydrocarbons in Drosophila melanogaster. J. Exp. Biol. 205: 3241-3249.

Savarit, F., Sureau, G., Cobb, M., and Ferveur, J.-F. 1999. Genetic elimination of known pheromones reveals the fundamental chemical basis of mating and isolation in Drosophila. Proc. Natl. Acad. Sci. 96: 9015-9020.

Siegel, R.W. and Hall, J.C. 1979. Conditioned responses in courtship behavior of normal and mutant Drosophila. Proc. Natl. Acad. Sci. 76: $3430-3434$.

Siwicki, K.K. and Ladewski, L. 2003. Associative learning and memory in Drosophila: Beyond olfactory conditioning. Behav. Proc. 64: 225-238.

Stocker, R.F. 1994. The organization of the chemosensory system in Drosophila melanogaster: A review. Cell Tissue Res. 275: 3-26.

Stocker, R.F. and Gendre, N. 1989. Courtship behavior of Drosophila genetically or surgically deprived of basiconic sensilla. Behav. Genet. 19: 371-385.

Stockinger, P., Kvitsiani, D., Rotkopf, S., Tirian, L., and Dickson, B.J. 2005. Neural circuitry that governs Drosophila male courtship behavior. Cell 121: 795-807.

Sureau, G. and Ferveur, J.F. 1999. Co-adaptation of pheromone production and behavioural responses in Drosophila melanogaster males. Genet Res. 74: 129-137.

Thorne, N., Chromey, C., Bray, S., and Amrein, H. 2004. Taste perception and coding in Drosophila. Curr. Biol. 14: 1065-1079.

Tompkins, L., Gross, A.C., Hall, J.C., Gailey, D.A., and Seigel, R.W. 1982. The role of female movement in the sexual behavior of Drosophila melanogaster. Behav. Genet. 12: 295-307.

Tompkins, L., Siegel, R.W., Gailey, D.A., and Hall, J.C. 1983. Conditioned courtship in Drosophila and its mediation by association of chemical cues. Behav. Genet. 13: 565-578.

Tully, T. and Quinn, W.G. 1985. Classical conditioning and retention in normal and mutant Drosophila melanogaster. J. Comp. Physiol. A 157: 263-277.

Vaias, L.J., Napolitano, L.M., and Tompkins, L. 1993. Identification of stimuli that mediate experience-dependent modification of homosexual courtship in Drosophila melanogaster. Behav. Genet. 23: 91-97.

Waddell, S. and Quinn, W.G. 2001. What can we teach Drosophila? What can they teach us? Trends Genet. 17: 719-726.

$\mathrm{Xu}$, A., Park, S.-K., D’Mello, S., Kim, E., Wang, Q., and Pikielny, C.W. 2002. Novel genes expressed in subsets of chemosensory sensilla on the front legs of male Drosophila melanogaster. Cell Tissue Res. 307: 381-392.

Zawistowski, S. 1988. A replication demonstrating reduced courtship of Drosophila melanogaster by associative learning. J. Comp. Psychol. 102: 174-176.

Received August 23, 2004; accepted in revised form August 23, 2005. 


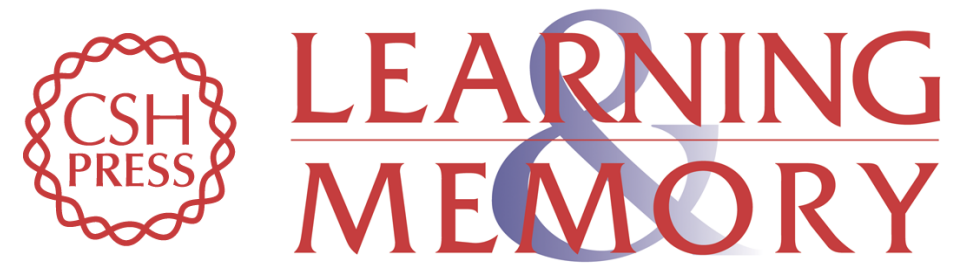

\section{The role of cuticular pheromones in courtship conditioning of Drosophila males}

Kathleen K. Siwicki, Paul Riccio, Lisa Ladewski, et al.

Learn. Mem. 2005, 12:

Access the most recent version at doi:10.1101/lm.85605

References This article cites 43 articles, 9 of which can be accessed free at:

http://learnmem.cshlp.org/content/12/6/636.full.html\#ref-list-1

License

Email Alerting Receive free email alerts when new articles cite this article - sign up in the box at the Service top right corner of the article or click here. 